from runs with stationary phases other than liquid paraffin. Peaks corresponding to methyl, propane and butane were detected on all four columns, while propane was detected on all except the oxydipropionitrile column.

Apples are not unique among plant organs in producing a variety of hydrocarbons. Dormant potatoes have recently been shown to yield a similar range of compounds (Burton, W. G., and Meigh, D. F., unpublished results). Whether the minute quantities of substances that are produced have any significant role in apple metabolism remains to be found.

Ditton Laboratory, D. F. Mrigh

Food Investigation Organization,

Department of Scientific and Industrial Research, Maidstone, Kent.

${ }^{1}$ McWilliam, I. G., and Dewar, R. A., Nature, 181, 760 (1958). Lovelock, J. E., J. Chromatogr., 1, 35 (1958). Meigh, D. F., J. Sci. Food Agric., 7, 396 (1956).

\section{Maintenance of Semipermeability of Plant Cell Membranes in the Absence of Metabolic Energy Supply}

Ir is generally believed that the semipermeability of cell membranes is closely associated with the living functions of the cell. According to Harvey" ". . . Selective permeability ... becomes the surest test to distinguish the living from the dead, holding where all others fail. It can truly be said of living cells that by their membrane ye shall know them."

In the results to be presented below we would like to show:

(1) That under certain special conditions cells can maintain their semipermeable properties while their energy-supply is drastically cut down, or even when all the energy-supplying processes are abolished and the cells can be regarded as non-living;

(2) That these special conditions are to some extent connected with electrostatic phenomena.

The experiments were carried out with thin slices of red beet root (Beta vulgaris) 200-400 $\mu$ thick, corresponding to $\mathbf{1}-3$ layers of cells. The slices were washed in aerated tap water for at least 24 hours. The degree of semipermeability of the membrane was estimated by following the appearance of red pigment in the external solution. Most of the results reported here, as will be seen, were all-or-none effects. In order that the external solution in the case of control samples should be completely colourless only freshly dug beetroots were employed.

When the slices were placed in $0.01 M$ sodium fluoride solution under a nitrogen atmosphere, the red pigment started to leak out after 7-9 hours, and after 24 hours there was no pigment left in the tissue. Slices thus treated for $\mathbf{2 4}$ hours did not evolve detectable amounts of carbon dioxide when placed in Warburg respirometers at $30^{\circ} \mathrm{C}$., and thus according to the present state of our knowledge were without energy supply. Now, if magnesium nitrate or sulphate in concentration of $0.1 M$ was present in the medium together with the sodium fluoride from the start of the experimental period, no leakage was observed even after 7 days. No evolution of carbon dioxide could be detected under these conditions. (Exactly similar results were obtained if, in addition to sodium fluoride, the medium contained $10^{-2} M$ sodium cyanide and $5 \times 10^{-4} M 2,4$-dinitrophenol.) If the slices were at this stage transferred to higher concentrations of the salts, for example, $0 \cdot 4 M$, plasmolysis could be observed under the microscope. A return to the turgid state was brought about by replacing the $0.4 M$ solution with $0.1 M$ solution. The semipermeability of the membranes of 'non-living' cells had thus been maintained in the presence of the magnesium salts. If these slices were transferred to distilled water instantaneous leakage occurred.

Further experiments investigated whether leakage could be prevented by the presence of other salts. Sodium chloride, sulphate and iodide were found to be efficient at concentrations of about $0.6-0.8 M$. Since some divalent cations are precipitated by sodium fluoride, this substance had to be omitted in subsequent experiments. Prolonged anærobiosis alone, however, caused considerable leakage and this could be entirely prevented by the addition of many salts, for example, calcium, lead and cobalt nitrates or manganese sulphate, etc., all in concentrations of $0 \cdot 1 \mathrm{M}$.

2,4-dinitrophenol, the best known uncoupler of phosphorylation, also induces leakage of red pigment. When the inhibitor was applied in concentration of $5 \times 10^{-4} M$ at $p H 5$ and at $30^{\circ} \mathrm{C}$., leakage started after 4-6 hours. It could be entirely prevented by including $0 \cdot 1-0 \cdot 2$ sodium or potassium chlorides, or $0.05 M$ magnesium or cobalt nitrates, in the original media. If slices which had been treated with 2,4dinitrophenol for 12-16 hours, in the absence of salts, were rinsed and transferred to distilled water, leakage continued at about the same rate for several hours. But if they were transferred instead to the salt solutions indicated above the leakage ceased at once.

It is reasonable to conclude that changes in semipermeability are due to some reversible changes in the physical state of some macromolecular structure in the membrane. When energy is supplied by metabolism the non-leaky state is maintained. But this is also achieved by high concentrations of salts. A relevant property of these concentrated salt solutions may be the screening of charges. That implies that we are dealing with electrostatic phenomena. Another indication that this is the case is the effect of $p \mathrm{H}$ on the system. When slices were placed for 48 hours in a graded series of $0.02 M$ phosphate buffers, in the presence of $0.01 M$ sodium fluoride and under a nitrogen atmosphere, all the red pigment leaked from the tissues at all $p H$ 's from $2 \cdot 5$ to $5 \cdot 0$. Leakage was less complete at $p \mathrm{H}^{\prime} \mathrm{s} 6.0$ and 7.0 . At $p \mathrm{H}^{\prime} \mathrm{s} 8.0,9.0$ and 10.0 no leakage occurred. The maintenance of semipermeability at high $p \mathrm{H}$, contrasting with the heavy leakage at low $p \mathrm{H}$, may be interpreted as showing that when the structure is negatively charged the membrane is non-leaky, whereas when positively charged it leaks. It is premature to envisage a model for the mechanism by which the living cell controls the physical state of the membrane, but there are grounds for assuming that this is achieved by means of the direct action of adenosine triphosphate on some membrane component.

These experiments, and their interpretation, will be discussed more fully elsewhere.

\section{B. Ginzburg}

Department of Botany,

The Hebrew University, Jerusalem,

${ }^{1}$ Harvey, E. N., in foreword to 'The Permeability of Natural Membranes' by H. Davison and J. F. Danielli (Cambridge University Press, 1952). 